Check for updates

Cite this: RSC Adv., 2019, 9, 23142

Received 21st June 2019

Accepted 22nd July 2019

DOI: 10.1039/c9ra04655k

rsc.li/rsc-advances

\section{Modulation of the electronic property of hydrogenated 2D tetragonal Ge by applying external strain}

\begin{abstract}
Chunyan Xu, (iD ${ }^{a}$ Jing Zhang, ${ }^{a}$ Ming Guo and Lingrui Wang ${ }^{\star b}$
Inspired by the novel properties of a newly predicted two-dimensional (2D) tetragonal allotrope of Ge called 2D tetragonal $\mathrm{Ge}$, first-principles calculations have been performed to explore the stability, and structural and electronic properties of 2D tetragonal Ge via hydrogenation, and the effect of external strain on structural and electronic properties of hydrogenated 2D tetragonal $\mathrm{Ge}$ is considered. Our calculations reveal that the hydrogenated 2D tetragonal $\mathrm{Ge}, \alpha-\mathrm{GeH}$ and $\beta-\mathrm{GeH}$, are proved to be dynamically and thermally stable. Both $\alpha-\mathrm{GeH}$ and $\beta-\mathrm{GeH}$ are semiconductors with a direct band gap of $0.953 \mathrm{eV}$ and indirect band gap of $2.616 \mathrm{eV}$, respectively. When applying external strain from $-7 \%$ to $7 \%, \alpha-\mathrm{GeH}$ is more energetically stable than $\beta-\mathrm{GeH}$ around the equilibrium geometry, $\beta-\mathrm{GeH}$ is more stable than $\alpha$ $\mathrm{GeH}$ when external strains exceed a certain critical value, respectively. The direct band gap of $\alpha$-GeH reduces rapidly from $2.008 \mathrm{eV}$ to $0.036 \mathrm{eV}$ as external strain increases from $-7 \%$ to $7 \%$, while the indirect band gap of $\beta-\mathrm{GeH}$ is changed slightly. Our results reveal that $\alpha-\mathrm{GeH}$ and $\beta-\mathrm{GeH}$ can offer an intriguing platform for nanoscale device applications and spintronics.
\end{abstract}

\section{Introduction}

Following the successful preparation of graphene, ${ }^{\mathbf{1}}$ twodimensional (2D) materials, including silicene, ${ }^{2}$ germanene, ${ }^{3}$ hexagonal boron nitride, ${ }^{4}$ transition metal dichalcogenides (TMDs), ${ }^{5}$ and phosphorene, ${ }^{6}$ have attracted extensive attention due to their outstanding electronic, optical, thermal and mechanical properties. In analogy to graphene, it is theoretically predicted that other group IV elements can form stable 2D honeycomb structures, namely silicene and germanene, and they have been successfully synthesized on different substrates. ${ }^{2,3}$ More recently, a 2D tetragonal allotrope of $\mathrm{C}$ consisting of repeated square and octagonal rings was predicted to be stable. ${ }^{7}$ First-principles calculations have proposed that the 2D tetragonal allotrope of $\mathrm{Si}$ is a nodal line semimetal, and hydrogenation could induce a semimetal-semiconductor transition in this silicene allotrope. ${ }^{8}$ Also, 2D tetragonal Ge and tetragonal Sn are theoretically predicted to be nodal line semimetals which are identified by nontrivial $\mathbf{Z}_{2}$ invariant and topological edge states at the sample boundaries. ${ }^{9}$ Additionally, some tetragonal graphenelike analogues, such group V elements, $\mathrm{XBi}(\mathrm{X}=\mathrm{Si}, \mathrm{Ge}, \mathrm{Sn})$ and TMDs, have also been proposed and predicted to be stable. ${ }^{10-16}$

ajilin Engineering Laboratory for Quantum Information Technology, Institute for Interdisciplinary Quantum Information Technology, Jilin Engineering Normal University, Changchun 130052, People's Republic of China

${ }^{b}$ Key Laboratory of Materials Physics of Ministry of Education, Department of Physics and Engineering, Zhengzhou University, Zhengzhou 450052, China. E-mail: wanglr@ zzu.edu.cn
It is crucial to open the band gap of 2D group IV materials for broadening the application in semiconductor devices, such as in power components and optoelectronic fields. Hydrogenation has been demonstrated to be one of the most accessible ways to modulate the properties of $2 \mathrm{D}$ materials. In this respect, it has been theoretically reported that hydrogenation significantly modifies the band properties and band gap of graphene, silicene and germanene, ${ }^{17}$ deriving them from semimetal state to a semiconductor state with a large band gap, and which have been synthesized in experiments. ${ }^{18-20}$ The band gap of 8-Pmmn borophene has been opened by hydrogenation..$^{21}$ The hydrogenation causes monolayer GaN to undergo an indirect to direct band gap transition. ${ }^{22}$ Generally speaking, external strain engineering is the other effective approach in modifying the electronic band structure of low dimensional materials. It is found that 2D materials, such as graphene, phosphorene and $\mathrm{MoS}_{2}$, can withstand a tensile strain of more than $20 \% .^{23-25} \mathrm{PbTe}$ films passivated with $\mathrm{H}$ atoms are semiconductors with a direct band gap of $0.015 \mathrm{eV}$, and the direct band gap can be tuned from $0.015 \mathrm{eV}$ to $0.065 \mathrm{eV}$ under external strain. ${ }^{26}$ The tensile strain can tune the band gap of GaN monolayer in the broad range of 4.44-2.27 eV. ${ }^{22}$ The $2 \mathrm{D}$ tetragonal XBi $(\mathrm{X}=\mathrm{Si}, \mathrm{Ge}, \mathrm{Sn})$ undergo a transition from a direct to indirect band gap or an indirect band gap semiconductor to metal by applying biaxial strain. ${ }^{16}$ It has been reported that the electronic property of monolayer $\mathrm{CrI}_{3}$ can be modified by applying strain, which undergoes a transition from magnetic-metal to half-metal to half-semiconductor to spin-relevant semiconductor as strain increases from $-15 \%$ to $10 \% .{ }^{27}$ The band gap of phosphorene 
experiences a direct-indirect-direct transition when axial strain is applied. ${ }^{23}$ It is indicated that compressive strain induces 1T$\mathrm{HfS}_{2}$ monolayer to undergo a semiconductor-metal transition. ${ }^{28}$

In the paper, we systematically investigate the stability, geometry structure and electronic property of 2D tetragonal Ge via hydrogenation, where two different atomic configurations are considered. We also explore the effect of external strain on the structural and electronic properties in the hydrogenated $2 \mathrm{D}$ tetragonal Ge.

\section{Computational details}

Our calculations were carried out based on the density functional theory in the Vienna $a b$ initio Simulation Package $(\mathrm{VASP})^{29-31}$ within the projector-augmented wave (PAW) method. ${ }^{32}$ The Perdew-Burke-Ernzerhof (PBE) of the generalized gradient approximation (GGA) ${ }^{33}$ was used as exchangecorrelation functional. The kinetic energy cutoff of the plane wave basis was set at $500 \mathrm{eV}$. The vacuum space more than $20 \AA$ was placed to avoid the interaction between the neighboring slabs. All structures were fully relaxed until the energy tolerances were smaller than $1 \times 10^{-6} \mathrm{eV}$ and the force on each atom was less than $0.01 \mathrm{eV}^{-1}$. The Brillouin zone was performed of a $13 \times 13 \times 1 k$-points. The HSE06 calculation was also used to check the validity of electronic band structure given by the PBE calculation. The spin-orbital-coupling (SOC) effect was considered in the calculation of the band structure. To examine the dynamic stability of the hydrogenated $2 \mathrm{D}$ tetragonal Ge, the phonon dispersions were performed by the finite displacement method in the PHONOPY code. ${ }^{34}$ The $a b$ initio molecular dynamics (AIMD) simulations were implemented to check the thermally stability at $300 \mathrm{~K}$ in the canonical (NVT), where the simulation time is limited to 10 ps with a time step of $1 \mathrm{fs}$. The binding energy was calculated to check the energetic stability, and the binding energy was defined by $E_{\mathrm{b}}=\left(E_{\mathrm{GeH}}-n E_{\mathrm{Ge}}-\right.$ $\left.n E_{\mathrm{H}}\right) / 2 n,{ }^{18}$ where $E_{\mathrm{GeH}}$ is the total energy of the hydrogenated 2D tetragonal Ge, $E_{\mathrm{Ge}}$ and $E_{\mathrm{H}}$ are the energies of the isolated Ge and $\mathrm{H}$ atoms, respectively, and $n$ is the number of Ge or $\mathrm{H}$ atom in the unit.

The applied external strain was defined as $\eta=\left(a-a_{0}\right) / a_{0}$, where $a_{0}$ is the lattice constant of the optimized strain-free structure, $a$ is the lattice constant of the strained systems, and just atomic position was allowed to relax for strained system. Negative and positive values of $\eta$ referred to compressive strain and tensile strain, respectively. The external strains in the range of $-7 \%$ to $7 \%$ with spacing of $1 \%$ were chosen in our calculations. In this work, two types of external strains, biaxial strain and uniaxial strain along the $x$ direction, were applied on $\alpha-\mathrm{GeH}$ and $\beta-\mathrm{GeH}$.

\section{Results and discussion}

Fig. 1 depicts the optimized atomic structures of two hydrogenated $2 \mathrm{D}$ tetragonal $\mathrm{Ge}$, denoted by $\alpha-\mathrm{GeH}$ with the hydrogen atoms alternate on both sides of the sheet and $\beta-\mathrm{GeH}$ with the hydrogen atoms alternate in pairs of the sheet. The optimized structural parameters of $\alpha-\mathrm{GeH}$ and $\beta-\mathrm{GeH}$, including lattice
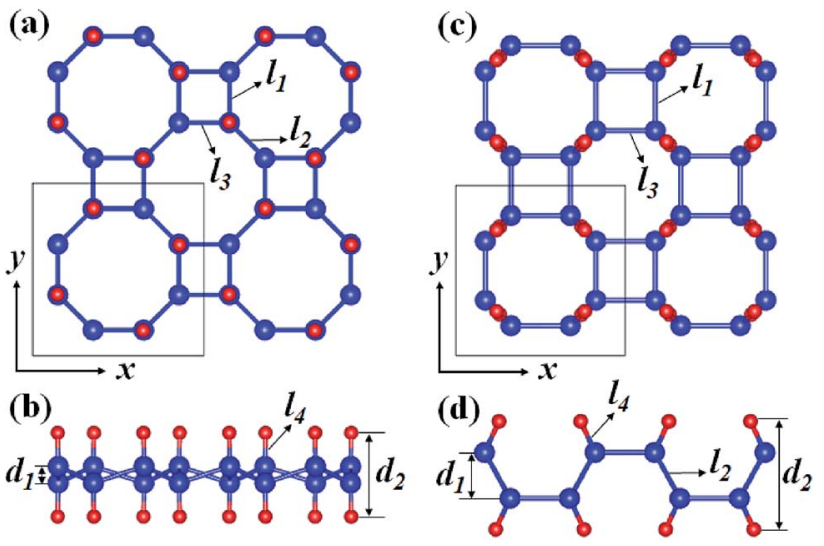

Fig. 1 Optimized geometries of (a) and (b) $\alpha-\mathrm{GeH}$ and (c) and (d) $\beta$ $\mathrm{GeH}$. The blue and red balls indicate $\mathrm{Ge}$ and $\mathrm{H}$ atoms, respectively.

constants $a_{0}$, Ge-Ge bond lengths $l_{1}$ and $l_{2}$, and the vertical distance between top and bottom $\mathrm{Ge}(\mathrm{H})$ layers $d_{1}\left(d_{2}\right)$, are summarized in Table 1. Except for the lattice constant of $\beta-\mathrm{GeH}$, all the corresponding parameters of $\alpha-\mathrm{GeH}$ and $\beta-\mathrm{GeH}$ are enlarged in comparison to pristine 2D tetragonal Ge. As listed in Table 1 . The calculated binding energies are $-2.832 \mathrm{eV}$ per atom and $\beta-\mathrm{GeH}-2.832 \mathrm{eV}$ per atom of $\alpha-\mathrm{GeH}$ and $\beta-\mathrm{GeH}$, respectively, indicating strong interactions between $\mathrm{H}$ atom and $\mathrm{Ge}$ atom. According to previous calculations, the binding energy of hydrogenated germanene is $-2.882 \mathrm{eV}$ per atom, ${ }^{17}$ and hydrogenated germanene has been synthesized in experiment, ${ }^{\mathbf{1 8}}$ it indicates that $\alpha-\mathrm{GeH}$ and $\beta-\mathrm{GeH}$ are stable and possible to experimentally obtained. Additionally, AIMD simulations are carried out at $300 \mathrm{~K}$ for $10 \mathrm{ps}$, and the total energies with respect to the simulation time and the final structure after simulations are displayed in Fig. 2(a). The total energies are fluctuated within a narrow range during simulations for both $\alpha-\mathrm{GeH}$ and $\beta$ $\mathrm{GeH}$, and the original structures can be nearly kept after the simulations, confirming the thermally stable of $\alpha-\mathrm{GeH}$ and $\beta$ $\mathrm{GeH}$. Furthermore, the phonon dispersions of $\alpha-\mathrm{GeH}$ and $\beta$ GeH are calculated and displayed in Fig. 2(b) and (c), there is no any imaginary frequency in the Brillouin zone, conforming the dynamic stability of $\alpha-\mathrm{GeH}$ and $\beta-\mathrm{GeH}$.

The electronic band structures of $\alpha-\mathrm{GeH}$ and $\beta-\mathrm{GeH}$ are calculated and plotted in Fig. 3. An energy gap opens in $2 \mathrm{D}$ tetragonal Ge by hydrogenation, $\alpha-\mathrm{GeH}$ and $\beta-\mathrm{GeH}$ are both semiconductors, the type of gap for hydrogenated $2 \mathrm{D}$ tetragonal Ge depends on its atomic configuration. It can be clearly seen

Table 1 The lattice constant $a_{0}, \mathrm{Ge}-\mathrm{Ge}$ bond lengths $l_{1}$ and $l_{2}\left(l_{3}\right), \mathrm{Ge}-$ $\mathrm{H}$ bond length $I_{4}$, and the vertical distance between top and bottom $\mathrm{Ge}(\mathrm{H})$ layers $d_{1}\left(d_{2}\right)$ shown in Fig. 1 , band gap $E_{\mathrm{g}}$, and binding energy $E_{\mathrm{b}}$ of $\alpha-\mathrm{GeH}$ and $\beta-\mathrm{GeH}$

\begin{tabular}{llllllllll}
\hline System & $a_{0}(\AA)$ & $l_{1}(\AA)$ & $\begin{array}{l}l_{2}\left(l_{3}\right) \\
(\AA)\end{array}$ & $l_{4}(\AA)$ & $d_{1}(\AA)$ & $d_{2}(\AA)$ & $E_{\mathrm{g}}(\mathrm{eV})$ & $\begin{array}{l}E_{\mathrm{b}}(\mathrm{eV} \\
\text { per atom })\end{array}$ \\
\hline $\mathrm{Ge}^{9}$ & 8.007 & 2.481 & 2.412 & - & 0.717 & - & 0.000 & -3.082 \\
$\alpha-\mathrm{GeH}$ & 8.073 & 2.493 & 2.455 & 1.564 & 0.737 & 3.865 & 0.953 & -2.832 \\
$\beta-\mathrm{GeH}$ & 7.184 & 2.492 & 2.455 & 1.564 & 1.870 & 4.535 & 2.343 & -2.830
\end{tabular}



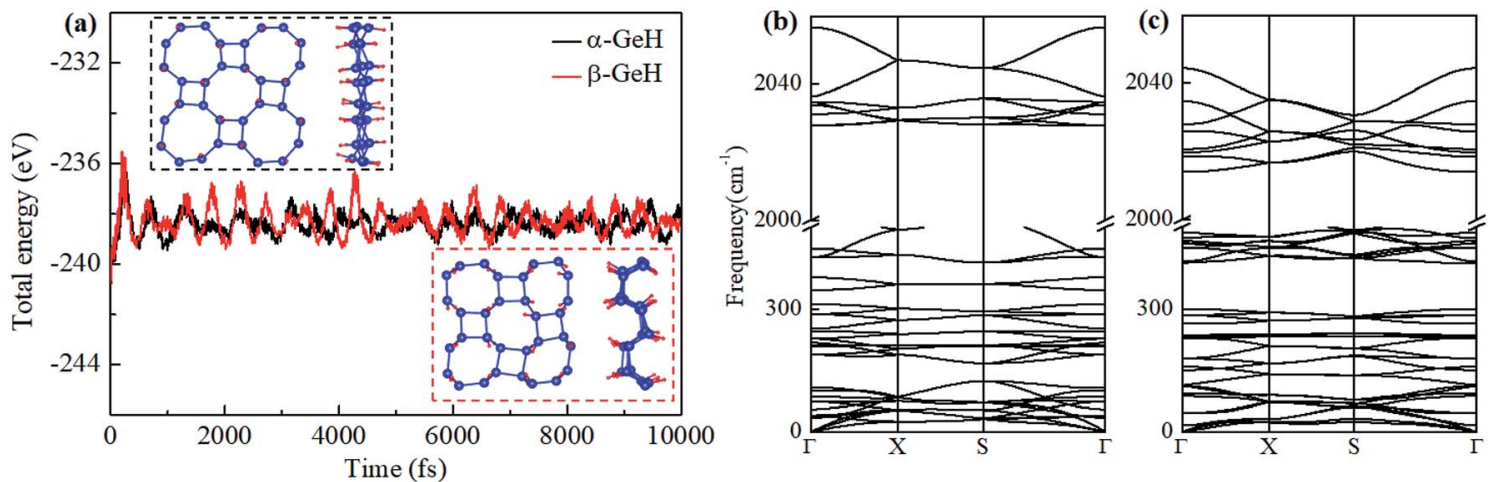

Fig. 2 (a) The total energy fluctuation of $\alpha-\mathrm{GeH}$ (black line) and $\beta-\mathrm{GeH}$ (red line), the insets are the final structures at the end of AIMD simulations. Phonon dispersions for (b) $\alpha-\mathrm{GeH}$ and (c) $\beta-\mathrm{GeH}$.

from Fig. 3, $\alpha-\mathrm{GeH}$ is direct band gap of $0.953 \mathrm{eV}$ with the valence band maximum (VBM) and conduction band minimum (CBM) at the $\Gamma$ point, while $\beta-\mathrm{GeH}$ is indirect band gap of $2.616 \mathrm{eV}$ with its VBM lying at $\Gamma$ point and CBM locating along the $S-Y$ direction. As shown in Fig. 3 , the VBM and CBM of $\alpha$ $\mathrm{GeH}$ and $\beta-\mathrm{GeH}$ are originated from the $\mathrm{p}_{x y}$ and s orbitals of Ge atom, respectively. In order to examine the effect of SOC, the electronic band structures of $\alpha-\mathrm{GeH}$ and $\beta-\mathrm{GeH}$ with
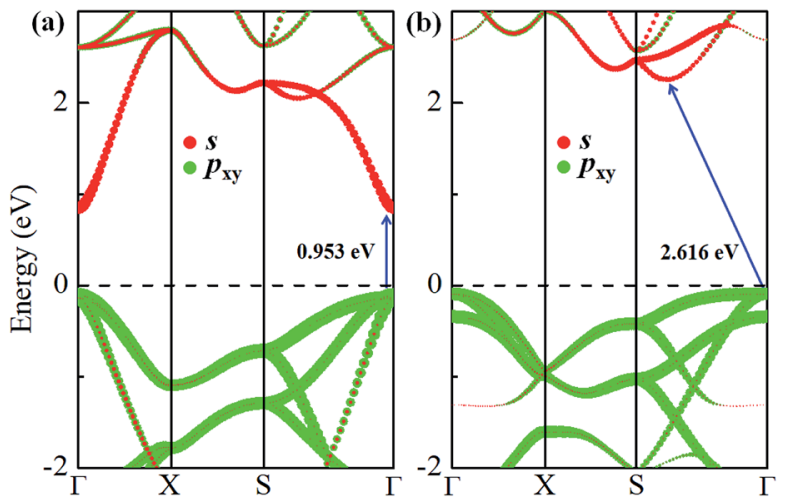

Fig. 3 Orbital-resolved band structures of unstrained (a) $\alpha-\mathrm{GeH}$ and (b) $\beta-\mathrm{GeH}$, red and green dots represent the contribution from $\mathrm{Ge}-\mathrm{p}_{x y}$ and $\mathrm{Ge}-\mathrm{s}$, respectively. The Fermi levels are set to zero.
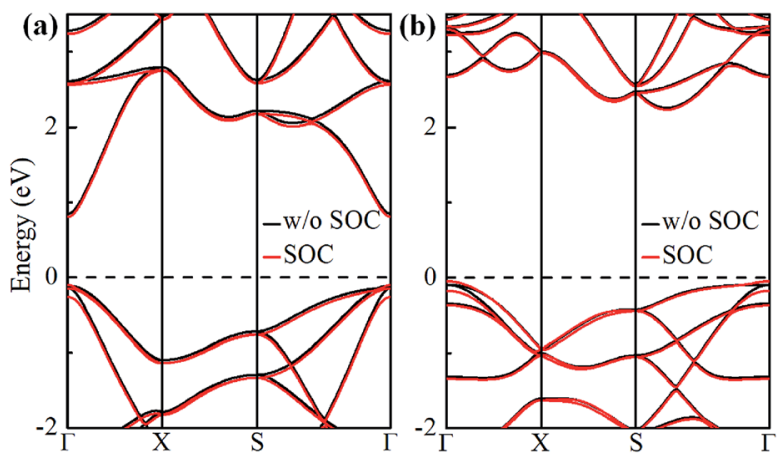

Fig. 4 Band structures of (a) $\alpha-\mathrm{GeH}$ and (b) $\beta-\mathrm{GeH}$ without SOC (black lines) and with SOC (red lines). The Fermi level is set at zero. considering the SOC effect are calculated and displayed in Fig. 4 . It can be seen from Fig. 4 that taking into account SOC, the band gaps of the unstrained $\alpha-\mathrm{GeH}$ and $\beta-\mathrm{GeH}$ is reduced slightly, which are changed to $0.904 \mathrm{eV}$ and $2.275 \mathrm{eV}$. Compared to band structures without SOC, there is no remarkable change in band gap and the shapes of the electronic structure near the Fermi level are essentially unchanged. Therefore, the effect of SOC on the electronic structure for $\alpha-\mathrm{GeH}$ and $\beta-\mathrm{GeH}$ are negligible. Then, the HSE calculation is used to check the electronic band structure of $\alpha-\mathrm{GeH}$ and $\beta-\mathrm{GeH}$, and the results are displayed in Fig. 5. It is clear that overall electronic band profiles calculated by PBE and HSE calculations are similar, the only difference is the calculated band gap, the HSE calculation enhance the band gap to $1.414 \mathrm{eV}$ and $3.036 \mathrm{eV}$ for $\alpha-\mathrm{GeH}$ and $\beta$ $\mathrm{GeH}$, respectively. Our work is mainly focused on the external strain effect on the band structure of $\alpha-\mathrm{GeH}$ and $\beta-\mathrm{GeH}$, and the HSE calculation could give results of the strain effects on the band structures consistent with that of the PBE calculation. The PBE calculation can able to correctly predict the trends of external strain effect on the electronic band structure and band gap for $\alpha-\mathrm{GeH}$ and $\beta$-GeH.

Recently, both experimental and theoretical studies have investigated that the electronic structure can be modulated by applying external strain. Here, we discuss the effect of external strain on the geometry structure and electronic band structures
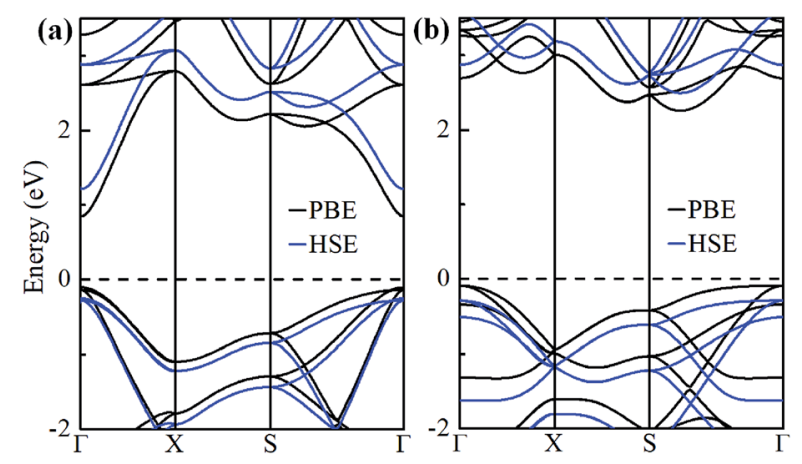

Fig. 5 Band structures of (a) $\alpha-\mathrm{GeH}$ and (b) $\beta-\mathrm{GeH}$ by using PBE (black lines) and HSE (blue lines) calculations. The Fermi level is set at zero. 

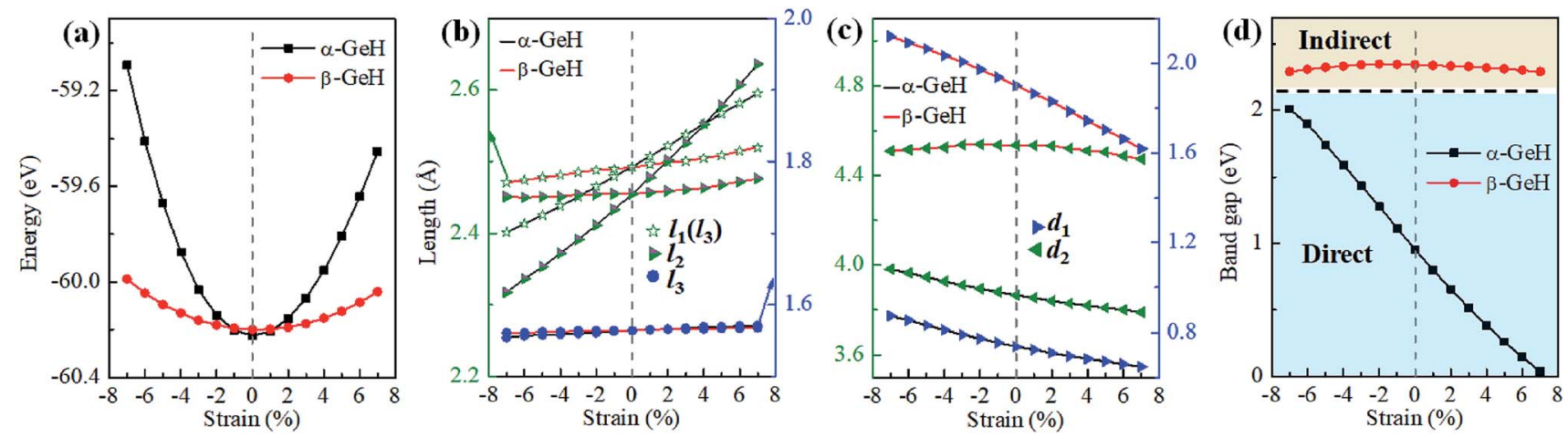

Fig. 6 The variations of (a) energies, (b) bond lengths, (c) vertical distances, and (d) band gaps of $\alpha-\mathrm{GeH}$ and $\beta$-GeH as a function of biaxial strains The direct and indirect band gap regions are highlighted with blue and orange, respectively.

of $\alpha-\mathrm{GeH}$ and $\beta-\mathrm{GeH}$, and the applied external strains ranged from $-7 \%$ to $7 \%$. First, we study the case of applying biaxial strain, the total energies with respect to biaxial strain for $\alpha-\mathrm{GeH}$ and $\beta-\mathrm{GeH}$ are calculated and the results can be seen from Fig. 6(a) $\alpha-\mathrm{GeH}$ is more energetically stable than $\beta$-GeH under strains in the range $\pm 2 \%$, while $\beta$-GeH is more stable than $\alpha$ GeH when strains larger than $\pm 2 \%$. Furthermore, the detailed analysis of the geometric structure for strained $\alpha-\mathrm{GeH}$ and $\beta$ GeH are also carried out. In Fig. 6(b), it can be clearly seen that the bond lengths $l_{1}$ and $l_{2}$ enhance linearly for $\alpha-\mathrm{GeH}$ under biaxial strains from $-7 \%$ to $7 \%$, while those of $\beta-\mathrm{GeH}$ display a very slightly increase, the bond length $l_{3}$ is hardly affected by biaxial strain and maintains a constant about $1.564 \AA$ for both $\alpha$ $\mathrm{GeH}$ and $\beta-\mathrm{GeH}$. When applying biaxial strains from $-7 \%$ to $7 \%$, the interlayer distance $d_{1}$ of $\alpha$ - and $\beta-\mathrm{GeH}$ and $d_{2}$ of $\alpha-\mathrm{GeH}$ decrease monotonously, the interlayer distance $d_{2}$ of $\beta-\mathrm{GeH}$ changes little as biaxial strain increases in Fig. 6(c).

Hence, the electronic band structure of $\alpha-\mathrm{GeH}$ and $\beta-\mathrm{GeH}$ under biaxial strains between $-7 \%$ and $7 \%$ are investigated, the results are displayed in Fig. 7. As can be seen from Fig. 7(a) and (b), $\alpha$-GeH is always remaining direct band gap character, the CBM at the $\Gamma$ point of $\alpha-\mathrm{GeH}$ shifts downward in a linear way and the VBM at the $\Gamma$ point shifts upward very slowly under biaxial strains of $-7 \%$ to $7 \%$. As displayed in Fig. $7(\mathrm{c})$ and (d), $\beta$ -
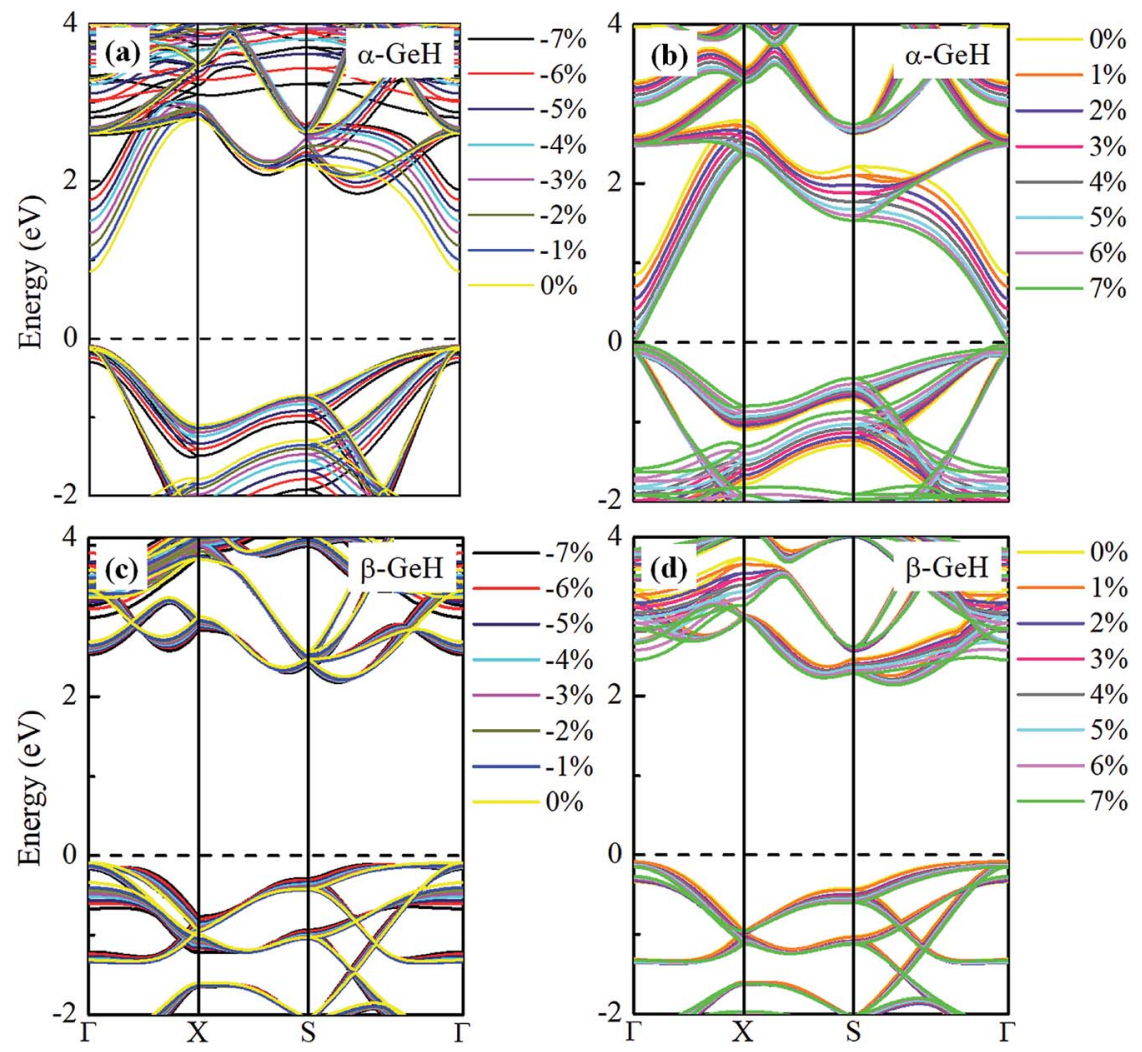

Fig. 7 Electronic band structures of (a) and (b) $\alpha-\mathrm{GeH}$, and (c) and (d) $\beta$-GeH under different biaxial strains. The Fermi levels are set to zero. 

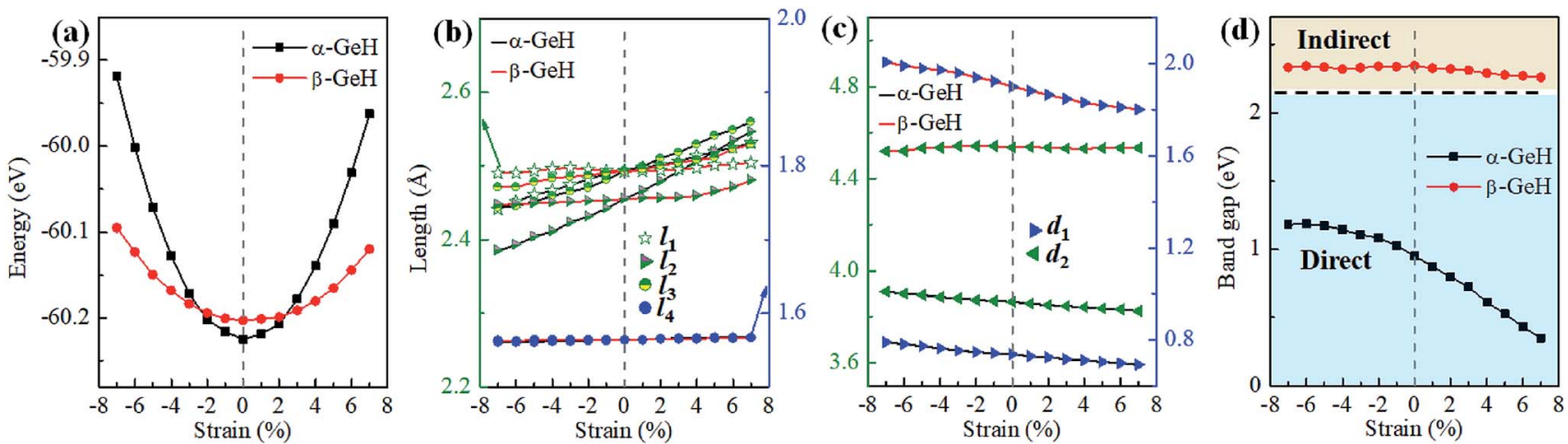

Fig. 8 The variations of (a) energies, (b) bond lengths, (c) vertical distances, and (d) band gaps of $\alpha$-GeH and $\beta$-GeH as a function of uniaxial strains. The direct and indirect band gap regions are highlighted with blue and orange, respectively.

GeH keeps indirect band gap property, the location of the VBM and CBM is almost unaffected within the biaxial strains range of $-7 \%$ to $7 \%$. It can been seen in Fig. $6(\mathrm{~d})$, the direct band gap of $\alpha-\mathrm{GeH}$ decreases sharply as the strain increases in the range from $-7 \%$ to $7 \%$, which change from $2.008 \mathrm{eV}$ to $0.036 \mathrm{eV}$, while the band gap of $\beta-\mathrm{GeH}$ changes slightly. The variation of band gap for $\alpha-\mathrm{GeH}$ and $\beta$-GeH also reflects the changes in their structure. In Fig. 6(b), the distance between the upper Ge atom and the lower Ge atom (bond lengths $l_{1}$ and $l_{2}$ ) increases linearly as strain increases, causing the bond between them to become weaker and weaker, therefore, the band gap of $\alpha-\mathrm{GeH}$ is gradually reduced. For $\beta-\mathrm{GeH}$, the bond lengths $l_{1}$ and $l_{2}$ varies slowly under biaxial strain, leading to little change in the band gap of $\beta-G e H$. Then, the uniaxial strain effects on geometric and electronic properties of $\alpha-\mathrm{GeH}$ and $\beta-\mathrm{GeH}$ are also studied, and the calculation results are shown in Fig. 8 and 9. As mentioned above, the tendency in energies, bond lengths, vertical distances and band gaps of $\alpha-\mathrm{GeH}$ and $\beta-\mathrm{GeH}$ is similar to that of applying biaxial strain. It can be seen from Fig. $8(\mathrm{a}), \alpha-\mathrm{GeH}$ is more energetically stable than $\beta$-GeH under strains in the range $\pm 3 \%$, while $\beta$-GeH is more stable than $\alpha$-GeH with strains larger than $\pm 3 \%$. When applying uniaxial strains from $-7 \%$ to $7 \%$, the band gap of $\alpha-\mathrm{GeH}$ can be tuned from $1.181 \mathrm{eV}$ to $0.349 \mathrm{eV}$, as plotted in Fig. 8(d). Our calculations show that $\alpha-\mathrm{GeH}$ and $\beta$ -
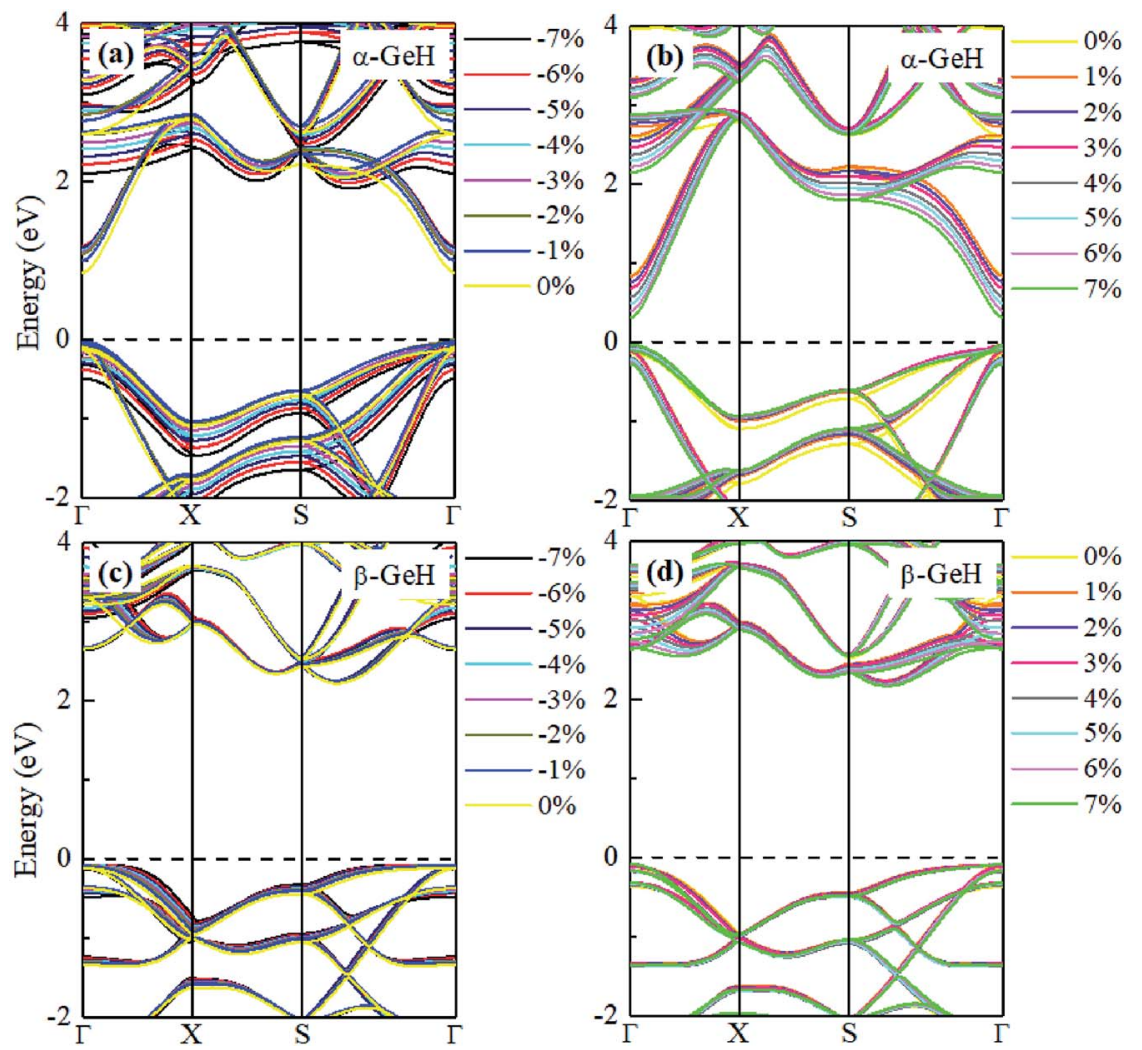

Fig. 9 Electronic band structures of (a) and (b) $\alpha-\mathrm{GeH}$, and (c) and (d) $\beta-\mathrm{GeH}$ under different uniaxial strains. The Fermi levels are set to zero. 
$\mathrm{GeH}$ remain their direct or indirect behavior under the biaxial and uniaxial strains and the band gap of $\alpha-\mathrm{GeH}$ can be tuned in a wide range.

\section{Conclusions}

In summary, based on first-principles calculations, we systemically study the stability, structural and electronic properties of 2D tetragonal Ge by hydrogenation, and the influence of external strain on the structural and electronic properties of the hydrogenated 2D tetragonal Ge is also investigated. Our calculated results indicate that $\alpha-\mathrm{GeH}$ is a direct semiconductor with a band gap of $0.953 \mathrm{eV}$, while $\beta-\mathrm{GeH}$ is an indirect semiconductor with a band gap of $2.616 \mathrm{eV}$. Applying external strains from $-7 \%$ to $7 \%, \alpha-\mathrm{GeH}$ is more energetically stable than $\beta-\mathrm{GeH}$ around the equilibrium geometry, $\beta-\mathrm{GeH}$ is more stable than $\alpha$ $\mathrm{GeH}$ when external strains exceed a certain critical value, respectively. The band gap of $\alpha-\mathrm{GeH}$ can be effectively tuned by applying external strain, which changes from $2.008 \mathrm{eV}$ to $0.036 \mathrm{eV}$ when the applied external strain varies from $-7 \%$ to $7 \%$, while the band gap of $\beta-\mathrm{GeH}$ is insensitive to external strain. $\alpha-\mathrm{GeH}$ and $\beta-\mathrm{GeH}$ possess excellent electronic property, showing great potential in nano-electric devices and spintronics.

\section{Conflicts of interest}

There are no conflicts to declare.

\section{Acknowledgements}

This work was supported by the Specialized Fund for the Doctoral Research of Jilin Engineering Normal University under Grants No. BSKJ201904, the China Postdoctoral Science Foundation (2019M652560), the postdoctoral research grant in Henan Province (1902013) and the Startup Research Fund of Zhengzhou University (32211173).

\section{References}

1 A. K. Geim and K. S. Novoselov, Nat. Mater., 2007, 6, 183.

2 B. Feng, Z. Ding, S. Meng, Y. Yao, X. He, P. Cheng, L. Chen and K. Wu, Nano Lett., 2012, 12, 3507-3511.

3 M. E. Dávila, L. Xian, S. Cahangirov, A. Rubio and G. Le Lay, New J. Phys., 2014, 16, 095002.

4 L. Song, L. Ci, H. Lu, P. B. Sorokin, C. Jin, J. Ni, A. G. Kvashnin, D. G. Kvashnin, J. Lou, B. I. Yakobson and P. M. Ajayan, Nano Lett., 2010, 10, 3209-3215.

5 Q. H. Wang, K. Kalantar-Zadeh, A. Kis, J. N. Coleman and M. S. Strano, Nat. Nanotechnol., 2012, 7, 699.

6 L. Li, Y. Yu, G. J. Ye, Q. Ge, X. Ou, H. Wu, D. Feng, X. H. Chen and Y. Zhang, Nat. Nanotechnol., 2014, 9, 372.

7 Y. Liu, G. Wang, Q. Huang, L. Guo and X. Chen, Phys. Rev. Lett., 2012, 108, 225505.

8 H. Wu, Y. Qian, Z. Du, R. Zhu, E. Kan and K. Deng, Phys. Lett. A, 2017, 381, 3754-3759.
9 C. Xu, Y. Wang, R. Han, H. Tu and Y. Yan, New J. Phys., 2019, 21, 033005.

10 Y. Zhang, J. Lee, W.-L. Wang and D.-X. Yao, Comput. Mater. Sci., 2015, 110, 109-114.

11 K. Liangzhi, T. Xin, M. Yandong, T. Hassan, Z. Liujiang, S. Ziqi, A. Du, C. Changfeng and C. S. Sean, 2D Mater., 2015, 2, 045010.

12 F. Ersan, E. Aktürk and S. Ciraci, Phys. Rev. B: Condens. Matter Mater. Phys., 2016, 94, 245417.

13 Y. Sun, C. Felser and B. Yan, Phys. Rev. B: Condens. Matter Mater. Phys., 2015, 92, 165421.

14 S. M. Nie, Z. Song, H. Weng and Z. Fang, Phys. Rev. B: Condens. Matter Mater. Phys., 2015, 91, 235434.

15 Y. Ma, L. Kou, X. Li, Y. Dai, S. C. Smith and T. Heine, Phys. Rev. B: Condens. Matter Mater. Phys., 2015, 92, 085427.

16 C. Xu, M. Zhu, J. Zhang, W. Wang and Y. Yan, Comput. Mater. Sci., 2018, 150, 314-320.

17 J. Kang, J. Li, F. Wu, S.-S. Li and J.-B. Xia, J. Phys. Chem. C, 2011, 115, 20466-20470.

18 E. Bianco, S. Butler, S. Jiang, O. D. Restrepo, W. Windl and J. E. Goldberger, ACS Nano, 2013, 7, 4414-4421.

19 J. Qiu, H. Fu, Y. Xu, A. I. Oreshkin, T. Shao, H. Li, S. Meng, L. Chen and K. Wu, Phys. Rev. Lett., 2015, 114, 126101.

20 D. C. Elias, R. R. Nair, T. M. G. Mohiuddin, S. V. Morozov, P. Blake, M. P. Halsall, A. C. Ferrari, D. W. Boukhvalov, M. I. Katsnelson, A. K. Geim and K. S. Novoselov, Science, 2009, 323, 610.

21 Z.-Q. Wang, T.-Y. Lü, H.-Q. Wang, Y. P. Feng and J.-C. Zheng, ACS Appl. Electron. Mater., 2019, 1, 667-674.

22 H. Shu, X. Niu, X. Ding and Y. Wang, Appl. Surf. Sci., 2019, 479, 475-481.

23 X. Peng, Q. Wei and A. Copple, Phys. Rev. B: Condens. Matter Mater. Phys., 2014, 90, 085402.

24 A. Castellanos-Gomez, M. Poot, G. A. Steele, H. S. J. van der Zant, N. Agraït and G. Rubio-Bollinger, Nanoscale Res. Lett., 2012, 7, 233.

25 K. S. Kim, Y. Zhao, H. Jang, S. Y. Lee, J. M. Kim, K. S. Kim, J.-H. Ahn, P. Kim, J.-Y. Choi and B. H. Hong, Nature, 2009, 457, 706.

26 Z. Huang and D. Zhang, J. Phys.: Condens. Matter, 2019, 31, 295503.

27 Z. Wu, J. Yu and S. Yuan, Phys. Chem. Chem. Phys., 2019, 21, 7750-7755.

28 N. Wu, X. Zhao, X. Ma, Q. Xin, X. Liu, T. Wang and S. Wei, Phys. E, 2017, 93, 1-5.

29 G. Kresse and J. Hafner, Phys. Rev. B: Condens. Matter Mater. Phys., 1994, 49, 14251-14269.

30 G. Kresse and J. Hafner, Phys. Rev. B: Condens. Matter Mater. Phys., 1993, 47, 558-561.

31 G. Kresse and J. Furthmüller, Phys. Rev. B: Condens. Matter Mater. Phys., 1996, 54, 11169-11186.

32 P. E. Blöchl, Phys. Rev. B: Condens. Matter Mater. Phys., 1994, 50, 17953-17979.

33 J. P. Perdew, K. Burke and M. Ernzerhof, Phys. Rev. Lett., 1996, 77, 3865-3868.

34 D. Alfè, Comput. Phys. Commun., 2009, 180, 2622-2633. 